\title{
On the projectivity of finitely generated flat modules
}

\author{
A. TARIZADEH \\ Department of Mathematics, Faculty of Basic Sciences, University of Maragheh \\ P.O. Box 55136-553, Maragheh, Iran \\ ebulfez1978@gmail.com
}

Received October 21, 2018 (revised July 18, 2019)

Presented by Juan B. Sancho

Accepted September 9, 2019

\begin{abstract}
In this paper, the projectivity of a finitely generated flat module of a commutative ring is studied through its exterior powers and invariant factors and then various new results are obtained. Specially, the related results of Endo, Vasconcelos, Wiegand, Cox-Rush and Puninski-Rothmaler on the projectivity of finitely generated flat modules are generalized.

Key words: exterior power, invariant factor, projectivity, S-ring, specialization cone, generalization cone.
\end{abstract}

AMS Subject Class. (2010): 13C10, 19A13, 13C11, 13E99.

\section{INTRODUCTION}

The main purpose of the present paper is to investigate the projectivity of finitely generated flat modules of a commutative ring. This topic has been the main subject of many articles in the literature over the years and it is still of current interest, see e.g. [3, 5, 6, 7, 11, 12, 13. Note that in general there are finitely generated flat modules which are not projective, see Example 2.9, also see [4, Tag $00 \mathrm{NY}$ ] as another example (note that our example is so simple than the cited one; it is also applicable for other purposes). The main motivation to investigate the projectivity of finitely generated flat modules essentially originates from the fact that every finitely generated flat module over a local ring is free, see Theorem 2.2. This result together with Theorem 2.8 play a major role in this paper.

In this paper, the projectivity of a finitely generated flat module of a commutative ring is studied through its exterior powers and invariant factors and then we obtain various new and interesting results. One of the features of this study is that some major results in the literature on the projectivity of finitely generated flat modules are generalized. Specially, Theorem 3.1 generalizes [5, Theorem 1], Theorem 3.2 improves a little [12, Theorem 2.1], 
Theorem 3.3 generalizes [13, Theorem 2], [3, Proposition 2.3], it also generalizes [11, Proposition 5.5 and Corollary 5.6] in the commutative case, and finally Corollary 3.10 generalizes [13, Theorem 2]. In fact, Theorem 3.3 can be viewed as a generalization of all of the above mentioned results. This theorem is one of the main novel contributions of this paper and has many non-trivial consequences. Theorems 3.16, 3.18 and 3.22 are another interesting results of this paper.

For reading the present paper having a reasonable knowledge on the exterior powers of a module is necessary. In this paper, all rings are commutative.

\section{Preliminaries}

We need the following material in the next section.

Lemma 2.1. Let $M$ be a finitely generated $R$-module, let $I=\operatorname{Ann}_{R}(M)$ and let $S$ be a multiplicative subset of $R$. Then $S^{-1} I=\operatorname{Ann}_{S^{-1} R}\left(S^{-1} M\right)$.

Proof. It is well known and easy.

A projective $R$-module is also called $R$-projective. We also use a similar terminology for free and flat modules.

Unlike the Kaplansky theorem [8] which states that every projective module over a local ring is free, but this is not true for flat modules. For example, the field of rationals $\mathbb{Q}$ is $\mathbb{Z}_{\mathfrak{p}}$-flat but it is not $\mathbb{Z}_{\mathfrak{p}}$-free where $\mathfrak{p}$ is a non-zero prime ideal of the ring of integers $\mathbb{Z}$. In spite of this, in the finite case we have the following interesting result which can be considered it as the analogue of the Kaplansky theorem for flat modules.

TheOREM 2.2. Every finitely generated flat module over a local ring is free.

Proof. See [9, Theorem 7.10] or [4, Tag 00NZ].

Lemma 2.3. Let $\phi: R \rightarrow S$ be a morphism of rings and $M$ a finitely generated flat $R$-module. Then $\operatorname{Ann}_{S}\left(M \otimes_{R} S\right)=\operatorname{Ann}_{R}(M) S$.

Proof. It is a local property implied by Lemma 2.1 and Theorem 2.2.

Let $M$ be an $R$-module, $n \geq 2$ a natural number and let $J_{n}$ be the $R$ submodule of $M^{\otimes n}$ generated by the collection of pure tensors of the form 
$x_{1} \otimes \ldots \otimes x_{n}$ with $x_{i}=x_{j}$ for some $i \neq j$. The quotient $R$-module $M^{\otimes n} / J_{n}$ is called the $n$-th exterior power of $M$ and it is denoted by $\Lambda_{R}^{n}(M)$ or simply by $\Lambda^{n}(M)$ if there is no confusion on the base ring $R$. Write $\Lambda^{0}(M)=R$ and $\Lambda^{1}(M)=M$. The canonical $R$-multilinear map $\eta: M^{n} \rightarrow \Lambda^{n}(M)$ given by $\left(x_{1}, \ldots, x_{n}\right) \mapsto x_{1} \wedge \ldots \wedge x_{n}:=x_{1} \otimes \ldots \otimes x_{n}+J_{n}$ is clearly alternative. The $R$-module $\Lambda^{n}(M)$ together with the map $\eta$ satisfy in the following universal property. For each alternative $R$-multilinear map $\phi: M^{n} \rightarrow N$ then there exists a unique morphism of $R$-modules $\phi^{\prime}: \Lambda^{n}(M) \rightarrow N$ such that $\phi=\phi^{\prime} \circ \eta$.

If $M$ is a finitely generated $R$-module, then $\Lambda^{n}(M)$ is a finitely generated $R$-module. If $R \rightarrow S$ is a morphism of rings and $M$ is an $R$-module, then $\Lambda^{n}(M) \otimes_{R} S$ as $S$-module is canonically isomorphic to $\Lambda_{S}^{n}\left(M \otimes_{R} S\right)$. It is also well known that if $M$ is a projective (resp. flat) $R$-module, then for each natural number $n, \Lambda^{n}(M)$ is a projective (resp. flat) $R$-module.

If $M$ is a $R$-module then the $n$-th invariant factor of $M$, denoted by $I_{n}(M)$, is defined as the annihilator of the $n$-th exterior power of $M$, i.e., $I_{n}(M)=$ $\operatorname{Ann}_{R}\left(\Lambda^{n}(M)\right)$.

Remark 2.4. If $M$ is a finitely generated flat $R$-module then Theorem 2.2 leads us to a function $\psi:$ Spec $R \rightarrow \mathbb{N}=\{0,1,2, \ldots\}$ which is defined as $\mathfrak{p} \mapsto \operatorname{rank}_{R_{\mathfrak{p}}}\left(M_{\mathfrak{p}}\right)$. It is called the rank map of $M$. It is obvious that the rank map is continuous if and only if it is locally constant (i.e., for each prime ideal $\mathfrak{p}$ of $R$ then there exists an open neighborhood $U \subseteq \operatorname{Spec}(R)$ of that point such that $\operatorname{rank}_{R_{\mathfrak{q}}}\left(M_{\mathfrak{q}}\right)=\operatorname{rank}_{R_{\mathfrak{p}}}\left(M_{\mathfrak{p}}\right)$ for all $\left.\mathfrak{q} \in U\right)$. It is well known that $\operatorname{Supp}\left(\Lambda^{n}(M)\right)=\left\{\mathfrak{p} \in \operatorname{Spec}(R): \operatorname{rank}_{R_{\mathfrak{p}}}\left(M_{\mathfrak{p}}\right) \geq n\right\}$.

If $\phi: R \rightarrow S$ is a morphism of rings then the induced map $\operatorname{Spec}(S) \rightarrow$ $\operatorname{Spec}(R)$ given by $\mathfrak{p} \mapsto \phi^{-1}(\mathfrak{p})$ is denoted by $\phi^{*}$.

The Jacobson radical of a ring $R$ is denoted by $\mathfrak{J}(R)$.

An ideal $I$ of a ring $R$ is called a pure ideal if the canonical ring map $R \rightarrow R / I$ is a flat ring map. Pure ideals are quite interesting and play an important role in commutative and non-commutative algebra (for instance, in classifying Gelfand rings and their dual rings). An ideal $I$ of a ring $R$ is a pure ideal if and only if $I_{\mathfrak{p}}=0$ or $I_{\mathfrak{p}}=R_{\mathfrak{p}}$, for each prime ideal $\mathfrak{p}$ of $R$. Hence, $I$ is an idempotent ideal.

Theorem 2.5. An ideal $I$ of a ring $R$ is a pure ideal if and only if $\mathrm{Ann}(f)+$ $I=R$ for all $f \in I$.

Proof. It is a local property implied by Theorem 2.2 . 
COROLlary 2.6. Let $M$ be a finitely generated flat $R$-module with annihilator $I$. Then $I$ is a pure ideal.

Proof. It is a local property implied by Theorem 2.2 and Theorem 2.5 .

Lemma 2.7. The annihilator of a finitely generated projective module is generated by an idempotent element.

Proof. It is deduced from [2, p. 132, Proposition 3.1].

The following result is well known, see [1, Chap. II, $\S 5.2$, Théorème 1], [4, Tag $00 \mathrm{NX}$ ] and [12, Proposition 1.3]. As a contribution, we provide a new proof of this result.

Theorem 2.8. Let $M$ be a finitely generated flat $R$-module. Then the following are equivalent:

(i) $M$ is $R$-projective.

(ii) The invariant factors of $M$ are finitely generated ideals.

(iii) The rank map of $M$ is locally constant.

Proof. (i) $\Rightarrow$ (ii). It is well-known that $\Lambda^{n}(M)$ is a finitely generated projective $R$-module and so by Lemma 2.7, $I_{n}(M)$ is a principal ideal.

(ii) $\Rightarrow$ (iii). It suffices to show that the rank map of $M$ is Zariski continuous. By Corollary 2.6, $I_{n}(M)$ is an idempotent ideal. Thus there exists some $a \in I_{n}(M)$ such that $(1-a) I_{n}(M)=0$. Clearly $a=a^{2}$ and $I_{n}(M)=R a$. By Remark 2.4. $\psi^{-1}(\{n\})=\operatorname{Supp} N \cap\left(\operatorname{Spec}(R) \backslash \operatorname{Supp} N^{\prime}\right)$ where $N=\Lambda^{n}(M)$ and $N^{\prime}=\Lambda^{n+1}(M)$. But $\operatorname{Supp} N=\operatorname{Spec}(R) \backslash V(1-a)$. Moreover, $\operatorname{Supp} N^{\prime}=V\left(I_{n+1}(M)\right)$ since $N^{\prime}$ is a finitely generated $R$-module. Therefore $\psi^{-1}(\{n\})$ is an open subset of Spec $R$.

(iii) $\Rightarrow$ (i). Apply Theorem 2.2 and 4 , Tag $00 \mathrm{NX}]$.

In the following we give an example of a finitely generated flat module which is not projective. It should be noted that finding such examples of modules is not as easy as one may think at first.

ExAmple 2.9. Let $R=\prod_{i \geq 1} A$ be an infinite product of copies of a nonzero ring $A$ and let $I=\bigoplus_{i \geq 1} A$ which is an ideal of $R$. If $f=\left(f_{i}\right) \in I$, then there exists a finite subset $D$ of $\{1,2,3, \ldots\}$ such that $f_{i}=0$ for all 
$i \in\{1,2,3, \ldots\} \backslash D$. Clearly $f=f g$ where $g=\left(g_{i}\right) \in I$ with $g_{i}=1$ for all $i \in D$ and $g_{i}=0$ for all $i \in\{1,2,3, \ldots\} \backslash D$. Hence, $I$ is a pure ideal of $R$ (i.e., $R / I$ is a finitely generated flat $R$-module). If $R / I$ is $R$-projective then by Lemma 2.7. there exists a sequence $e=\left(e_{i}\right) \in R$ such that $I=R e$. Thus there exists a finite subset $E$ of $\{1,2,3, \ldots\}$ such that $e_{i}=0$ for all $i \in\{1,2,3, \ldots\} \backslash E$. Clearly $\{1,2,3, \ldots\} \backslash E \neq \emptyset$. Pick some $k \in\{1,2,3, \ldots\} \backslash E$. There is some $r=\left(r_{i}\right) \in R$ such that $\left(\delta_{i, k}\right)_{i \geq 1}=r e$ where $\delta_{i, k}$ is the Kronecker delta. In particular, $1_{A}=r_{k} e_{k}=r_{k} 0_{A}=0_{A}$. This is a contradiction. Therefore $R / I$ is not $R$-projective.

\section{Projectivity: MAin Results}

Throughout this section, $M$ is a finitely generated flat $R$-module.

The following technical result generalizes [5, Theorem 1].

Theorem 3.1. Let $R \rightarrow S$ be an injective ring map. Then $M$ is $R$-projective if and only if $M \otimes_{R} S$ is $S$-projective.

Proof. Let $M \otimes_{R} S$ be $S$-projective. Without loss of generality, we may assume that $R \subseteq S$ is an extension of rings. First we shall prove that $I=$ $\operatorname{Ann}_{R}(M)$ is a principal ideal. By Lemma 2.3, $I S=L$ where $L=\operatorname{Ann}_{S}(N)$ and $N=M \otimes_{R} S$. Hence by Lemma 2.7, there is an idempotent $e \in S$ such that $I S=S e$. Let $J=S(1-e) \cap R$. Clearly $I J=0$. We have $I+J=R$. If not, then there exists a prime ideal $\mathfrak{p}$ of $R$ such that $I+J \subseteq \mathfrak{p}$. Thus, by Theorem 2.2, $I_{\mathfrak{p}}=0$. Therefore the extension of $I S$ under the canonical map $S \rightarrow S \otimes_{R} R_{\mathfrak{p}}$ is zero. Thus there exists an element $s \in R \backslash \mathfrak{p}$ such that $s e=0$ and so $s=s(1-e)$. Hence $s \in J$. But this is a contradiction. Therefore $I+J=R$. It follows that there is an element $c \in I$ such that $c=c^{2}$ and $I=R c$. Now, let $n \geq 1$. We have $\Lambda^{n}(M)$ is a finitely generated flat $R$-module. Moreover, $\Lambda^{n}(M) \otimes_{R} S$ is $S$-projective because it is canonically isomorphic to $\Lambda_{S}^{n}\left(M \otimes_{R} S\right)$. Thus, by what we have proved above, $I_{n}(M)$ is a principal ideal. Hence, by Theorem 2.8, $M$ is $R$-projective. The reverse is easy and well known.

The following result is also technical and generalizes [12, Theorem 2.1].

THEOREM 3.2. Let $J$ be an ideal of $R$ which is contained in the Jacobson radical of $R$. If $M / J M$ is $R / J$-projective, then $M$ is $R$-projective. 
Proof. First we shall prove that $I=\operatorname{Ann}_{R}(M)$ is a principal ideal. By Lemma 2.3, $L=I+J$ where $L=\operatorname{Ann}_{R}(M / J M)$. Also, by Lemma 2.7. $\operatorname{Ann}_{R / J}(M / J M)=L / J$ is a principal ideal. This implies that $I=R x+I \cap J$ for some $x \in R$ since $L / J=(I+J) / J$ is canonically isomorphic to $I /(I \cap J)$. $I=R x$ : Let $\mathfrak{m}$ be a maximal ideal of $R$. By Theorem 2.2, $I_{\mathfrak{m}}$ is either the whole localization or the zero ideal. If $I_{\mathfrak{m}}=0$ then $(R x)_{\mathfrak{m}}=0$ since $R x \subseteq I$. If $I_{\mathfrak{m}}=R_{\mathfrak{m}}$ then $I$ is not contained in $\mathfrak{m}$. Thus $R x$ is also not contained in $\mathfrak{m}$ since $I \cap J \subseteq J \subseteq \mathfrak{m}$. Hence $(R x)_{\mathfrak{m}}=R_{\mathfrak{m}}$. Therefore $I=R x$. Now let $n \geq 1$ and let $N=\Lambda^{n}(M)$. Then $N / J N$ is $R / J$-projective, because $N / J N$ as $R / J$-module is canonically isomorphic to $\Lambda_{R / J}^{n}(M / J M)$ and $\Lambda_{R / J}^{n}(M / J M)$ is $R / J$-projective. But $N$ is a finitely generated flat $R$-module. Therefore, by what we have proved above, $I_{n}(M)=\operatorname{Ann}_{R}(N)$ is a principal ideal. Thus the invariant factors of $M$ are finitely generated ideals and so by Theorem 2.8, $M$ is $R$-projective.

Motivated by the Grothendieck's relative point of view, then we obtain the following result which (beside Theorems 2.2 and 2.8) is one of the most powerful results on the projectivity of finitely generated flat modules.

Theorem 3.3. Let $\phi: R \rightarrow S$ be a ring map whose kernel is contained in the Jacobson radical of $R$. Then $M$ is $R$-projective if and only if $M \otimes_{R} S$ is $S$-projective.

Proof. Let $M \otimes_{R} S$ be $S$-projective. Clearly $M / J M$ is a finitely generated flat $R / J$-module and $M / J M \otimes_{R / J} S \simeq M \otimes_{R} S$ is $S$-projective where $J=$ $\operatorname{Ker} \phi$. Moreover $R / J$ can be viewed as a subring of $S$ via $\phi$. Therefore, by Theorem 3.1, $M / J M$ is $R / J$-projective. Then by applying Theorem 3.2 , we get that $M$ is $R$-projective. The reverse is easy and well known.

The above theorem has many consequences.

Recall that a ring $R$ is called an $S$-ring (" $S$ " referes to Sakhajev) if every finitely generated flat $R$-module is $R$-projective.

Corollary 3.4. Let $\phi: R \rightarrow S$ be a ring map whose kernel is contained in the Jacobson radical of $R$. If $S$ is an $S$-ring then $R$ is as well.

Proof. If $M$ is a finitely generated flat $R$-module then $M \otimes_{R} S$ is a finitely generated flat $S$-module and so, by the hypothesis, it is $S$-projective. Therefore by Theorem $3.3, M$ is $R$-projective. 
CoROllary 3.5. If there exists a prime ideal $\mathfrak{p}$ of a ring $R$ such that the kernel of the canonical ring map $R \rightarrow R_{\mathfrak{p}}$ is contained in the Jacobson radical of $R$, then $R$ is an $S$-ring.

Proof. By Theorem 2.2, every local ring is an $S$-ring, then apply Corollary 3.4 .

CoROllary 3.6. If there exists a prime ideal $\mathfrak{p}$ of a ring $R$ such that the canonical ring map $R \rightarrow R_{\mathfrak{p}}$ is injective, then $R$ is an $S$-ring.

Proof. It is an immediate consequence of Corollary 3.5 .

Corollary 3.7. Every integral domain is an $S$-ring.

Proof. It is an immediate consequence of Corollary 3.6 ,

Corollary 3.8. If the Jacobson radical of a ring $R$ contains a prime ideal $\mathfrak{p}$ of $R$, then $R$ is an $S$-ring.

Proof. Clearly Ker $\pi \subseteq \mathfrak{p}$ where $\pi: R \rightarrow R_{\mathfrak{p}}$ is the canonical ring map. Therefore by Corollary $3.5, R$ is an $S$-ring.

Another proof. By Corollary $3.7, R / \mathfrak{p}$ is an $S$-ring. Thus by Corollary 3.4 , $R$ is an $S$-ring.

Remark 3.9. Let $S$ be a subset of a ring $R$. The polynomial ring $R\left[x_{s}: s \in\right.$ $S$ ] modulo $I$ is denoted by $S^{(-1)} R$ where the ideal $I$ is generated by elements of the form $s x_{s}^{2}-x_{s}$ and $s^{2} x_{s}-s$ with $s \in S$. We call $S^{(-1)} R$ the pointwise localization of $R$ with respect to $S$. Amongst them, the pointwise localization of $R$ with respect to itself, namely $R^{(-1)} R$, has more interesting properties; for further information please consult with [10]. Note that Wiegand [13] utilizes the notation $\widehat{R}$ instead of $R^{(-1)} R$. Clearly $\eta(s)=\eta(s)^{2}\left(x_{s}+I\right)$ and $x_{s}+$ $I=\eta(s)\left(x_{s}+I\right)^{2}$ where $\eta: R \rightarrow S^{(-1)} R$ is the canonical map and the pair $\left(S^{(-1)} R, \eta\right)$ satisfies in the following universal property: "for each such pair $(A, \phi)$, i.e. $\phi: R \rightarrow A$ is a ring map and for each $s \in S$ there is some $c \in A$ such that $\phi(s)=\phi(s)^{2} c$ and $c=\phi(s) c^{2}$, then there exists a unique ring map $\psi: S^{(-1)} R \rightarrow A$ such that $\phi=\psi \circ \eta "$. Now let $\mathfrak{p}$ be a prime ideal of $R$ and consider the canonical map $\pi: R \rightarrow \kappa(\mathfrak{p})$ where $\kappa(\mathfrak{p})$ is the residue field of $R$ at $\mathfrak{p}$. By the above universal property, there is a (unique) ring map $\psi: S^{(-1)} R \rightarrow \kappa(\mathfrak{p})$ such that $\pi=\psi \circ \eta$. Thus $\eta$ induces a surjection between the corresponding spectra. This, in particular, implies that the kernel 
of $\eta$ is contained in the nilradical of $R$. Using this, then the following result generalizes [13, Theorem 2].

Corollary 3.10. If there exists a subset $S$ of $R$ such that $M \otimes_{R} S^{(-1)} R$ is $S^{(-1)} R$-projective, then $M$ is $R$-projective.

Proof. It is an immediate consequence of Theorem 3.3 .

In what follows we get some new results essentially based on the referee's excellent comments.

Corollary 3.11. Let $\phi: R \rightarrow S$ be a morphism of rings such that the induced map $\phi^{*}$ has the dense image. Then $M$ is $R$-projective iff $M \otimes_{R} S$ is $S$-projective.

Proof. It is an immediate consequence of Theorem 3.3 , because from equality $\overline{\operatorname{Im} \phi^{*}}=\operatorname{Spec}(R)$ we get that the kernel of $\phi$ is contained in $\mathfrak{J}(R)$, the Jacobson radical of $R$.

Lemma 3.12. Let $\left\{R_{k}, \mathfrak{m}_{k}\right\}_{k}$ be a family of local rings. Then the kernel of the canonical ring map $\pi: R=\prod_{k} R_{k} \rightarrow \prod_{k} R_{k} / \mathfrak{m}_{k}$ is the Jacobson radical of $R$.

Proof. If the sequence $x=\left(x_{k}\right) \in R$ is a member of Ker $\pi$ then $x_{k} \in \mathfrak{m}_{k}$ for all $k$. To prove $x \in \mathfrak{J}(R)$ it suffices to show $1+x y$ is invertible in $R$ for all $y=\left(y_{k}\right) \in R$. For each $k$, there exists some $z_{k} \in R_{k}$ such that $\left(1+x_{k} y_{k}\right) z_{k}=1$ because $R_{k}$ is a local ring. It follows that $(1+x y) z=1$ where $z=\left(z_{k}\right)$. Conversely, let $x \in \mathfrak{J}(R)$. For each $k$, then $\mathfrak{M}_{k}:=\pi_{k}^{-1}\left(\mathfrak{m}_{k}\right)$ is a maximal ideal of $R$ because the ring map $R / \mathfrak{M}_{k} \rightarrow R_{k} / \mathfrak{m}_{k}$ induced by the canonical projection $\pi_{k}: R \rightarrow R_{k}$ is an isomorphism. Therefore $x \in \mathfrak{M}_{k}$ for all $k$.

Corollary 3.13. Let $X \subseteq \operatorname{Spec}(R)$ be a subset. Then the following statements are equivalent:

(i) $M \otimes_{R} S$ is $S$-projective where $S=\prod_{\mathfrak{p} \in X} \kappa(\mathfrak{p})$.

(ii) $M \otimes_{R} S^{\prime}$ is $S^{\prime}$-projective where $S^{\prime}=\prod_{\mathfrak{p} \in X} R / \mathfrak{p}$.

(iii) $M \otimes_{R} S^{\prime \prime}$ is $S^{\prime \prime}$-projective where $S^{\prime \prime}=\prod_{\mathfrak{p} \in X} R_{\mathfrak{p}}$. 
If moreover $\bigcap_{\mathfrak{p} \in X} \mathfrak{p} \subseteq \mathfrak{J}(R)$, then the above statements are equivalent with the following:

(iv) $M$ is $R$-projective.

Proof. It is an immediate consequence of Theorem 3.3 .

The subsets $\operatorname{Min}(R)$ and $\operatorname{Max}(R)$ are typical examples which satisfy the hypothesis of Corollary 3.13 .

Corollary 3.14. Consider the following commutative diagram of rings

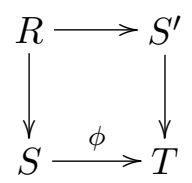

in which the kernel of $\phi$ is contained in the Jacobson radical of $S$. If $M \otimes_{R} S^{\prime}$ is $S^{\prime}$-projective, then $M \otimes_{R} S$ is $S$-projective.

Proof. If $M \otimes_{R} S^{\prime}$ is $S^{\prime}$-projective then it is easy to see that

$$
\left(M \otimes_{R} S^{\prime}\right) \otimes_{S^{\prime}} T \simeq M \otimes_{R} T \simeq\left(M \otimes_{R} S\right) \otimes_{S} T
$$

is $T$-projective. But $M \otimes_{R} S$ is a finitely generated flat $S$-module. Therefore by Theorem 3.3, $M \otimes_{R} S$ is $S$-projective.

Definition 3.15. If $X$ is a subset of $\operatorname{Spec}(R)$ then we call $\bigcup_{\mathfrak{p} \in X} V(\mathfrak{p})$ the specialization cone of $X$ and it is denoted by $X_{s}$. Dually, we call $\bigcup_{\mathfrak{p} \in X} \Lambda(\mathfrak{p})$ the generalization cone of $X$ and it is denoted by $X_{g}$ where $\Lambda(\mathfrak{p})=\{\mathfrak{q} \in$ $\operatorname{Spec}(R): \mathfrak{q} \subseteq \mathfrak{p}\}$.

Theorem 3.16. Let $X \subseteq \operatorname{Spec}(R)$ be a subset. Put $S:=\prod_{\mathfrak{p} \in X} \kappa(\mathfrak{p})$ and $S^{\prime}:=\prod_{\mathfrak{p} \in X_{s}} \kappa(\mathfrak{p})$. Then $M \otimes_{R} S$ is $S$-projective iff $M \otimes_{R} S^{\prime}$ is $S^{\prime}$-projective.

Proof. Consider the canonical injective ring map $T=\prod_{\mathfrak{p} \in X} R / \mathfrak{p} \rightarrow S$. Then by Theorem 3.1, $M \otimes_{R} S \simeq\left(M \otimes_{R} T\right) \otimes_{T} S$ is $S$-projective iff $M \otimes_{R} T$ is $T$-projective. By the axiom of choice, we obtain a function $\sigma: X_{s} \rightarrow X$ such 
that $\sigma(\mathfrak{p}) \subseteq \mathfrak{p}$ for all $\mathfrak{p} \in X_{s}$ and $\sigma(\mathfrak{p})=\mathfrak{p}$ for all $\mathfrak{p} \in X$. For each $\mathfrak{p} \in X$, consider the canonical injective ring map $R / \mathfrak{p} \rightarrow \prod_{\mathfrak{q} \in \sigma^{-1}(\mathfrak{p})} R / \mathfrak{q}$. Then we get the canonical injective ring map $T \rightarrow T^{\prime}=\prod_{\mathfrak{p} \in X_{s}} R / \mathfrak{p}$. Again by Theorem 3.1. $M \otimes_{R} T$ is $T$-projective iff $M \otimes_{R} T^{\prime}$ is $T^{\prime}$-projective. Similarly above, by applying Theorem 3.1 to the canonical injective ring map $T^{\prime} \rightarrow S^{\prime}$, we get that $M \otimes_{R} T^{\prime}$ is $T^{\prime}$-projective iff $M \otimes_{R} S^{\prime}$ is $S^{\prime}$-projective.

Corollary 3.17. Let $\mathfrak{p}$ be a prime ideal of a ring $R$ and put $S:=$ $\prod_{\mathfrak{q} \in V(\mathfrak{p})} \kappa(\mathfrak{q})$. Then $M \otimes_{R} S$ is $S$-projective.

Proof. It is an immediate consequence of Theorem 3.16 .

Theorem 3.18. Let $X \subseteq \operatorname{Spec}(R)$ be a subset. Put $S:=\prod_{\mathfrak{p} \in X} \kappa(\mathfrak{p})$ and $S^{\prime}:=\prod_{\mathfrak{p} \in X_{g}} \kappa(\mathfrak{p})$. Then $M \otimes_{R} S$ is $S$-projective iff $M \otimes_{R} S^{\prime}$ is $S^{\prime}$-projective.

Proof. The kernel of the canonical ring map $T=\prod_{\mathfrak{p} \in X} R_{\mathfrak{p}} \rightarrow S$ is the Jacobson radical of $T$, see Lemma 3.12 . Therefore by Theorem $3.3, M \otimes_{R} S \simeq$ $\left(M \otimes_{R} T\right) \otimes_{T} S$ is $S$-projective if and only if $M \otimes_{R} T$ is $T$-projective. By the axiom of choice, there exists a function $\sigma: X_{g} \rightarrow X$ such that $\mathfrak{p} \subseteq \sigma(\mathfrak{p})$ for all $\mathfrak{p} \in X_{g}$ and $\sigma(\mathfrak{p})=\mathfrak{p}$ for all $\mathfrak{p} \in X$. For each $\mathfrak{p} \in X$, consider the canonical injective ring map $R_{\mathfrak{p}} \rightarrow \prod_{\mathfrak{q} \in \sigma^{-1}(\mathfrak{p})} R_{\mathfrak{q}}$. Then we get the canonical injective ring map $T \rightarrow T^{\prime}=\prod_{\mathfrak{p} \in X_{g}} R_{\mathfrak{p}}$. Thus by Theorem 3.1. $M \otimes_{R} T$ is $T$-projective iff $M \otimes_{R} T^{\prime}$ is $T^{\prime}$-projective. Again by Lemma 3.12, the kernel of the canonical ring map $T^{\prime} \rightarrow S^{\prime}$ is contained in the Jacobson radical of $T^{\prime}$. Hence by Theorem 3.3, $M \otimes_{R} T^{\prime}$ is $T^{\prime}$-projective if and only if $M \otimes_{R} S^{\prime}$ is $S^{\prime}$-projective.

Corollary 3.19. Let $\mathfrak{p}$ be a prime ideal of a ring $R$ and put $S:=$ $\prod_{\mathfrak{q} \in \Lambda(\mathfrak{p})} \kappa(\mathfrak{q})$. Then $M \otimes_{R} S$ is $S$-projective.

Proof. It is an immediate consequence of Theorem 3.18 . 
Corollary 3.20. Let $X \subseteq Y \subseteq \operatorname{Spec}(R)$ be two subsets such that either $Y \subseteq X_{s}$ or $Y \subseteq X_{g}$. Put $S:=\prod_{\mathfrak{p} \in X} \kappa(\mathfrak{p})$ and $S^{\prime}:=\prod_{\mathfrak{p} \in Y} \kappa(\mathfrak{p})$. Then $M \otimes_{R} S$ is $S$-projective iff $M \otimes_{R} S^{\prime}$ is $S^{\prime}$-projective.

Proof. If $Y \subseteq X_{s}$ then $X_{s}=Y_{s}$, so apply Theorem 3.16 in this case. But if $Y \subseteq X_{g}$ then $X_{g}=Y_{g}$, and so apply Theorem 3.18.

Lemma 3.21. Let $\phi: R \rightarrow S$ be a morphism of rings and put $S^{\prime}:=$ $\prod_{\mathfrak{p} \in \operatorname{Im} \phi^{*}} \kappa(\mathfrak{p})$. Then $M \otimes_{R} S$ is $S$-projective if and only if $M \otimes_{R} S^{\prime}$ is $S^{\prime}$-projective.

Proof. If $\mathfrak{p} \in \operatorname{Im} \phi^{*}$ and $\mathfrak{q} \in\left(\phi^{*}\right)^{-1}(\mathfrak{p})$ then we have the canonical ring map $\kappa(\mathfrak{p}) \rightarrow \kappa(\mathfrak{q})$ which is injective since every ring map from a field into a non-zero ring is injective. Then we get the canonical injective ring map $\kappa(\mathfrak{p}) \rightarrow \prod_{\mathfrak{q} \in\left(\phi^{*}\right)^{-1}(\mathfrak{p})} \kappa(\mathfrak{q})$. So we get the canonical injective ring map $S^{\prime} \rightarrow T=$ $\prod_{\mathfrak{q} \in \operatorname{Spec}(S)} \kappa(\mathfrak{q})$ which fits in the following commutative diagram

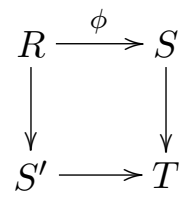

where the unnamed arrows are the canonical morphisms. It is easy to see that the kernel of the canonical morphism $S \rightarrow T$ is the nilradical of $S$ which is contained in the Jacobson radical of $S$. Then the assertion is deduced by twice using of Corollary 3.14 .

Given a subset $X \subseteq \operatorname{Spec}(R)$, denote $X^{(1)}:=\left(X_{s}\right)_{g}$ and $X_{(1)}:=\left(X_{g}\right)_{s}$, and inductively $X^{(n)}:=\left(X^{(n-1)}\right)^{(1)}$ and $X_{(n)}:=\left(X_{(n-1)}\right)_{(1)}$. Note that in general, $X^{(n)} \neq X_{(n)}$. For example, if $X=\{2 \mathbb{Z}\} \subseteq \operatorname{Spec}(\mathbb{Z})$ then $X^{(1)}=\{0,2 \mathbb{Z}\}$ but $X_{(1)}=\operatorname{Spec}(\mathbb{Z})$.

Theorem 3.22. Let $\phi: R \rightarrow S$ be a morphism of rings and $X=\operatorname{Im} \phi^{*}$. Assume there exists some $n \geq 1$ such that $\bigcap_{\mathfrak{p} \in X^{(n)}} \mathfrak{p}$ is contained in the Jacobson radical of $R$. If $M \otimes_{R} S$ is $S$-projective then $M$ is $R$-projective. 
Proof. By Lemma 3.21, $M \otimes_{R} S$ is $S$-projective iff $M \otimes_{R} S^{\prime}$ is $S^{\prime}$ - projective where $S^{\prime}:=\prod_{\mathfrak{p} \in X} \kappa(\mathfrak{p})$. By the successive applications of Theorems 3.16 and 3.18 , eventually after finite times we get that $M \otimes_{R} S^{\prime}$ is $S^{\prime}$-projective iff $M \otimes_{R} T$ is $T$-projective where $T=\prod_{\mathfrak{p} \in X^{(n)}} \kappa(\mathfrak{p})$. But the kernel of the canonical ring map $R \rightarrow T$ is equal to $\bigcap_{\mathfrak{p} \in X^{(n)}}^{\mathfrak{p} \in X}$. Thus by Theorem 3.3, $M$ is $R$-projective.

Theorem 3.23. Let $\phi: R \rightarrow S$ be a morphism of rings and $X=\operatorname{Im} \phi^{*}$. Suppose there exists some $n \geq 1$ such that $\bigcap_{\mathfrak{p} \in X_{(n)}} \mathfrak{p}$ is contained in the Jacobson radical of $R$. If $M \otimes_{R} S$ is $S$-projective, then $M$ is $R$-projective.

Proof. It is proven exactly like Theorem 3.22 .

\section{ACKNOWLEDGEMENTS}

The author would like to give sincere thanks to the referee for very careful reading of the paper and for his/her very valuable and excellent suggestions and comments which greatly improved the paper. I would also like to thank Professors Wolmer Vasconcelos and Jesús M.F. Castillo for various scientific correspondences during the writing of this paper.

\section{REFERENCES}

[1] N. Bourbaki, "Algèbre Commutative", Chapitres 1 à 4, Springer, Berlin, 2006.

[2] H. Cartan, S. Eilenberg, "Homological Algebra", Princeton University Press, Princeton, N. J., 1956.

[3] S.H. Cox JR., R.L. Pendleton, Rings for which certain flat modules are projective, Trans. Amer. Math. Soc. 150 (1970), 139-156.

[4] A.J. DE Jong ET AL., Stacks Project. http://stacks.math.columbia.edu

[5] S. Endo, On flat modules over commutative rings, J. Math. Soc. Japan 14(3) (1962), 284-291.

[6] A. FACChini ET AL., Finitely generated flat modules and a characterization of semiperfect rings, Comm. Algebra 31 (9) (2003), 4195-4214.

[7] S. Jondrup, On finitely generated flat modules, Math. Scand. 26 (1970), $233-240$.

[8] I. Kaplansky, Projective modules, Ann. of Math. 68 (1958), 372-377.

[9] H. Matsumura, "Commutative Ring Theory", Cambridge University Press, Cambridge, 1989. 
[10] J.P. OLIVIER, Anneaux absolument plats universels et épimorphismes à buts réduits, in "Séminaire Samuel. Algèbre Commutative", Tomme 2 (6) (19671968), $1-12$.

[11] G. Puninski, P. Rothmaler, When every finitely generated flat module is projective, J. Algebra 277 (2004), 542-558.

[12] W. Vasconcelos, On finitely generated flat modules, Trans. Amer. Math. Soc. 138 (1969), 505-512.

[13] R. WiEgAnd, Globalization theorems for locally finitely generated modules, Pacific J. Math. 39 (1) (1971), 269-274. 
